\title{
Password-manager friendly (PMF): Semantic annotations to improve the effectiveness of password managers
}

\author{
Frank Stajano, Max Spencer, Graeme Jenkinson, Quentin Stafford-Fraser \\ \{frank.stajano, max.spencer, graeme.jenkinson, \\ quentin.stafford-fraser\}@cl.cam.ac.uk \\ University of Cambridge Computer Laboratory \\ 15 JJ Thomson Avenue, Cambridge, CB3 0FD, United Kingdom
}

\begin{abstract}
Subtle and sometimes baffling variations in the implementation of password-based authentication are widespread on the web. Despite being imperceptible to end users, such variations often require that password managers implement complex heuristics in order to act on the user's behalf. These heuristics are inherently brittle. As a result, password managers are unnecessarily complex and yet they still occasionally fail to work properly on some websites. In this paper we propose PMF, a specification of simple semantic labels for password-related web forms. These semantic labels allow a software agent such as a password manager to extract meaning, such as which site the login form is for and what field in the form corresponds to the username. Our spec also allows the agent to generate a strong password on the user's behalf. PMF reduces a password manager's dependency on complex heuristics, making its operation more effective and dependable and bringing usability and security advantages to users and website operators.
\end{abstract}

\section{Introduction}

We don't have to explain to this audience that, on the web, we are asked to remember way too many passwords. One reasonable way of coping with this burden is with a password manager - a piece of software that remembers passwords on the user's behalf and submits them automatically when required. All modern browsers such as Chrome, Firefox and Internet Explorer provide an integrated password manager. Because websites frequently have slight differences in the way they handle asking the user to type a password (or to define a new one), every password manager must implement complex heuristics in order to parse, auto-fill and submit the password-requesting web pages. Such code is inherently fragile and requires continuous maintenance as login web pages evolve and become fancier. As a result, some websites don't work seamlessly with password managers.

Password managers would be simpler and more dependable if websites adopted a set of semantic labels for HTML forms that allowed unambiguous registration 
and submission of passwords by programs acting on the user's behalf. In this paper we offer two main contributions. First, we document the many ways in which websites ask for passwords and the many subtle ways in which the heuristics commonly employed by password managers can break, demonstrating how such code requires extensive maintenance to be reliable. Second, and most important, we propose PMF, a practical set of semantic labels that websites may immediately adopt. We also very briefly discuss incentives and benefits for the various parties involved.

\section{Inconsistencies in password-based login on the web}

Ignoring issues of style and presentation, password-based authentication on the web presents a fairly consistent interface to the user. To log in, users first find the login form, enter their username and password for that site into the appropriate boxes, and then press return or click the submit button. And, to a first approximation, the behaviour of the browser and the website is consistent across sites as well: the username and password entered into the form are sent to the server in an HTTPS POST request and a session cookie is returned. However, when we look in more detail, we notice a huge range of variations, some subtle and some baffling. Whilst these variations are imperceptible to the user, they present difficulties for a software agent parsing or automatically submitting the login form. This is because sub-tasks like entering the right username and password "into the appropriate boxes" are non-trivial and must rely on heuristics.

One of the most common mistakes password managers make is offering to save incorrect passwords. The user makes an error when typing their password, but despite an error message on the resultant page, their password manager still creates a dialog box asking the user if they want to save that password. This is simply because there's no standard signal to tell the password manager that the login was unsuccessful; the web server typically returns a "200 OK" HTTP response because a page was successfully served. At the time of writing, both Firefox and Internet Explorer both offered to save an incorrect password when logging into facebook.com. However Chromium and Google Chrome did not. These browsers employ a heuristic: if the response from the server contains a form with the same destination as the one which was submitted, then assume the submission failed ${ }^{1}$. While we expect this works in many cases, we can still envisage cases where it may not; or, worse, where it may cause false negativesthat is, not offering to save a correct password.

It is difficult for a password manager to correctly identify the fields in an account creation form. Login forms typically have just two fields, one for the username and another for the password, both of which are important to the password manager. Account creation forms, however, may have many more, sometimes spread across multiple pages, and the password manager needs to

\footnotetext{
${ }^{1}$ See password_manager.cc in the Chromium source code (https://code.google. $\mathrm{com} / \mathrm{p} / \mathrm{chromium} /$ codesearch\#chromium/src/components/password_manager/ core/browser/password_manager.cc).
} 
know which of them contains the username, that is, the unique account identifier to be supplied alongside the password when logging in. We found that Firefox and Chrome would just save a password with no associated username when we filled out the amazon.co.uk account creation page. In the case of Chrome, a duplicate record, including the username, was saved after logging in for the first time.

Another source of many problems for password managers is JavaScript. Some sites, such as Pinterest [1], use JavaScript to insert the login form into the page dynamically, that is, after the raw HTML in the response has been rendered by the web browser. Besides being annoying for users who browse with JavaScript disabled for security reasons, this practice also complicates the task of a password manager. Instead of parsing the HTML document just once at load-time to find any login forms, it must also monitor all changes made to the Document Object Model (DOM) by JavaScript thereafter.

The sins of JavaScript don't end there, though. Many sites use JavaScript to actually submit the form, thereby confusing utilities such as password managers that commonly intercept submission of the form to save the credentials. Forgoing a simple submit input adds little benefit and obfuscates the login process. Furthermore, to mitigate Cross-Site Request Forgery (CSRF) attacks [2], JavaScript is sometimes used to insert values (nonces/challenges associated with the session) into hidden fields within the login form before submission. For example, the following hidden input is added to the form on the Vimeo login page (https://vimeo.com/log_in) using JavaScript:

<input type="hidden" name="token" value="8113..." />

Attempts to log in to Vimeo with JavaScript disabled fail. Programs that parse or submit a login form must be compatible with such approaches, without being explicitly aware of what they achieve or even that they are being used.

Another problem facing a software agent such as a password manager is extracting the meaning (semantics) from the HTML login form. In the first instance, we'd like to determine what site the login form is for. The continued prevalence of phishing attacks demonstrates that reliably determining the website of a login page is too difficult for many humans. Software agents should have an advantage here. Indexing the username and password by the site's URL ensures that, provided HTTPS is used, the username and password are only submitted to correct site. Unfortunately, things are rarely this simple. Some websites have login forms on multiple pages - for example Facebook has one on its main landing page (www.facebook.com) and one on a dedicated login page (www.facebook. com/login.php). Should these login forms be considered as being for the same service? In the case of Facebook, both URLs are in the same second level domain facebook.com, so the answer is probably yes. But what about in a corporate intranet, where diverse services such as for submitting expenses and time sheets are all likely to be under the same second level domain?

It might be argued that services should only be considered the same if they have exactly the same URL. But what about the query string? Does that have to match as well? What about the order of the query parameters? What about 
dynamic URLs that provide alternative but equivalent encodings of the URL's query component? Any heuristic trying to shed light on this morass is likely to get things wrong (at least some of the time). Should users really have to accept that the computer doesn't even know what service is being logged in to?

\section{Incentives}

Our proposal offers obvious advantages to users in terms of usability (you don't have to remember or type the passwords any more) and security ${ }^{2}$ (you can use strong, distinct passwords). The advantages for password manager writers are even clearer (without guesswork, code becomes simpler, more reliable and much easier to maintain). Let's thus spend a few words on the incentives for website operators.

We believe it is in the best interests of website operators to support password managers: the website users will gain in usability and security. If users, thanks to password managers, adopted strong unique random passwords, website operators would have much less to worry about confidentiality compromises of their hashed password file.

We understand that website operators don't want to allow bots to register thousands of accounts and we support this goal. Any techniques the websites may wish to use to ensure the presence of a human registrant (from CAPTCHAs to telephone callbacks and so forth) will continue to be available. We are only concerned with helping the human registrant store the password in a password manager instead of having to remember it in their brain. Only websites with delusions of grandeur may still believe that, regardless of all other demands on the user's memory and patience, their password is so important that it must be uncrackably strong and different from any others and never written down. They should study the Compliance Budget model [3], manage risks more maturely and cure their superiority complex.

\section{The PMF semantic markup}

\subsection{Overview}

We propose adding "password-manager friendly" (PMF) semantic markup ${ }^{3}$ to forms related to creating, accessing and managing user accounts, to simplify the following tasks:

- Finding forms and determining their purpose (login, registration, etc.).

\footnotetext{
${ }^{2}$ Users of password managers are still exposed to malware; we are not claiming that the security offered by password managers is absolute (see section 5). Besides, our proposal implicitly also supports higher-security password managers running on dedicated hardware.

3 The latest version (as well as a complete revision history) of the PMF specification can be found at https://github.com/pmfriendly/pmf-specification.
} 
- Finding the important inputs within the forms.

- Parsing password policies and generating valid new passwords.

- Detecting errors.

We adopt a simple and pragmatic approach used in other HTML microformats, of using semantic class names. A class attribute value can be specified for any HTML element [4] and the use of semantic class names is supported by the W3C [5]. We use the pmf prefix as a poor man's namespace to avoid clashes with programmer-defined class names. For example ${ }^{4}$, a login form is marked with the pmf-login class:

<form action="/login" method="POST" class="pmf-login" >

Although form inputs have other attributes such as name and type which may often give sufficient semantic information, standardised class values can be used to remove any ambiguity ${ }^{5}$. For example, not all inputs with type="password" are for long-term passwords: some are for one-time codes generated by hardware tokens. Furthermore, as name attribute values are sometimes automatically generated by web frameworks or are specified by other standards such as OAuth [7], use of these attributes could cause conflicts. In contrast, any HTML element may have multiple classes [4], so our use of semantic class names ensures interoperability.

\subsection{Forms}

Being able to reliably determine the type or purpose of a given form enables a software agent like a password manager to offer a richer and/or more consistent user experience. form elements should be marked with the semantic classes specified in Table 1.

Table 1. Semantic classes for forms.

\begin{tabular}{|l|l|}
\hline Form type & Semantic class name \\
\hline Login & pmf-login \\
Registration & pmf-registration \\
Change password & pmf-change-password \\
Password reset & pmf-reset-password \\
\hline
\end{tabular}

\subsection{Inputs}

Username Login and registration forms typically contain an input element of type text or email for entering a username (which is often the user's email address). These inputs should be marked with the pmf-username class:

\footnotetext{
${ }^{4}$ In these examples, grey highlights indicate PMF-related additions.

${ }^{5}$ In section 5 we discuss the specification of the autocomplete attribute from the HTML 5.1 working draft [6].
} 
Username or email address:

<input type="text" name="user" class="pmf-username" />

Password resets and changes are tricky for a password manager because the software cannot tell - in the case where a user may have multiple accounts with the same website - which password is being changed. For example, a simple experiment using Firefox's built-in password manager and two Google accounts reveals that, in some cases, the password manager must prompt the user to ask which account they are updating the password for, even though they are already logged in.

We propose that site authors should include a hidden-type field in these forms, marked with the pmf-username semantic class and with its value set to the username of the relevant account:

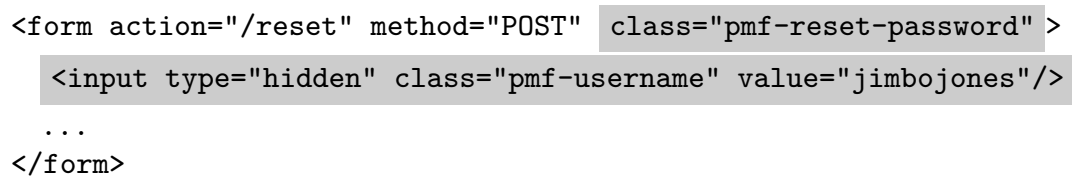

Passwords Inputs for passwords typically appear in all four of the above form types. Some password inputs, such as those in registration forms, are for new passwords, while others are for existing passwords. These sub-types are unambiguously distinguished by the pmf-new-password and pmf-password semantic classes respectively. It is useful to distinguish them because they appear together in "change password" forms. These typically contains three password-type inputs, one for the user's current password and two for their desired new password (one to confirm the other). All three will have a different name attribute values but, using semantic class names, the purpose of each input is made clear:

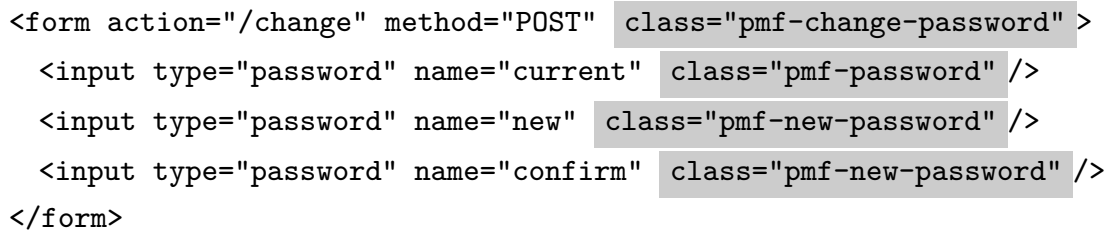

Stay signed in Many login forms include a "stay signed in" check box which allows the user to control whether their session with a website should persist across multiple browser sessions. If present, this input should be marked with the pmf-stay-signed-in class:

Stay signed in?

<input type="checkbox" name="persist" class="pmf-stay-signed-in" />

Annotating the "stay signed in" check box allows a software agent to apply a global policy on staying signed in for the user, across all websites. Many websites 
tick the "stay signed in" box by default and users accept this. But, if their password manager could apply a "never stay signed in" policy for them, they may be happy for it to do so and thereby gain a valuable security (and privacy) boost by not being permanently signed-in to their online accounts.

Another scenario in which this feature might be useful is the cybercafé: for the benefit of the patrons, the web browsers installed on the public cybercafé machines might be configured to disable the "stay signed in" feature by default.

Hidden inputs Forms often contain hidden-type input elements which are not visible when the HTML is rendered ${ }^{6}$. As human users are normally unaware of and cannot interact with these inputs, it is not useful for a software agent acting on the user's behalf to be able to interact with them either and we don't propose any additional markup.

\subsection{Password composition policies}

Large-scale password leaks have shown that many users optimise for memorability and typing convenience rather than security, choosing trivially-guessable passwords like 123456, qwerty or password. Password composition policies ("between 8 and 16 characters, of which at least one uppercase, one digit and one symbol") are an attempt to enforce generation of passwords that will be harder to guess. Aside from their debatable effectiveness, one further problem with these password policies is that websites do not offer them in machine-readable form, which makes it hard for a password manager to generate a strong password that is also compliant. A random machine-generated candidate password such as

\section{LAxwHNDNSECcZ2IiNnEPUT1R"SsoyQ3kt8AEJCeCtj jw5eLv6+XsnfW3,}

much stronger than any that a human would ever memorize or retype, would be rejected by many websites because it exceeds the maximum allowable length, or because it fails to include a character from the set $\$ \# \$ \% *$, or because it includes the disallowed character ", not to mention what websites would say about characters outside the ASCII range - the study by Bonneau and Xu [9] of passwords from users whose native language doesn't fit into ASCII is instructive.

In a previous version of this work we defined a simple specification for a machine-readable (JSON) description of a password composition policy, capable of expressing most commonly observed policies. Our intention was to avoid forcing unnecessary changes on the back-end: much as we disliked restrictions on password length ${ }^{7}$, we wanted webmasters to be able to make their website

\footnotetext{
${ }^{6}$ The values of these hidden inputs are usually populated by the web server when it generates the HTML of the page and then not changed on the client side. For example web frameworks, such as Django [8], use them to implement Cross Site Request Forgery protection.

${ }^{7}$ Often just an indication that the back-end is not even hashing the passwords, as observed by Bonneau and Preibusch [10].
} 
PMF-compliant without having to change anything about their password composition policy: merely adding a machine-readable description of their existing policy would be enough.

But we have since come to the conclusion that that strategy is sub-optimal. There is really no reason for the back-end to impose restrictions on password length on security grounds. If a password manager is generating and remembering a password on behalf of the user, it should be allowed to make the password as long and as complicated as it wishes. The password composition policy should only apply to passwords generated and remembered by humans; but passwords longer than a threshold $t$ (say 64 characters from the base-64 subset) should be considered as "non-human" and not subject to restrictions ${ }^{8}$. Sensible websites will not have length restrictions in their password composition policy anyway; those that do can become PMF-compliant simply by adding a conditional statement to the tune of

if length $>t$ then accept the password straight away.

This bold simplifying step from our side (PMF compliance requiring that passwords of unlimited length be allowed ${ }^{9}$ ) brings dramatic payoffs: it frees the webmaster from having to describe their policy in a machine-readable language (because humans can be served by the human-readable version and machines can just generate a sufficiently long random string) and it frees the password manager developer from having to write a parser for the policy.

The machine-generated passwords will be strong, random and too long to be memorized or transcribed: they will look similar to the one shown at the beginning of this section. For situations where users may need to access the website without being supported by their usual password manager, they are free to choose less complex passwords that can be memorized or at least transcribed. Such passwords will not fall into the "beyond $t$ characters" exclusion and will still have to comply with the website's traditional password composition policy intended for human-chosen secrets.

We believe that this is a better strategy than our previous one of making the policy machine-readable. By minimizing the amount of work that each party has

\footnotetext{
8 This is not to say that humans could never use passwords or passphrases of that length, or that passwords of that length are necessarily always unguessable. What we mean instead is that, once we agree that a competent and non-malicious agent is generating strong random passwords, once we go beyond $t$ characters then further checks are not necessary nor useful.

${ }^{9}$ In our latest PMF specification, besides always requiring the semantic markup described in the other sections, we define full PMF compliance as requiring that the policy accept passwords of length over $t$ regardless of their composition but we still grant partial compliance to websites that don't implement this exception. Partially PMF compliant sites still allow reliable automated interaction for login, even though they don't guarantee that the software agent will be able to define a compliant strong password.
} 
to do to become PMF-compliant ${ }^{10}$ we maximize the chance that PMF will be adopted.

\subsection{Errors}

As mentioned previously, determining whether a login attempt (or other action) was successful or not is a difficult problem for software agents, because at the HTTP layer a "200 OK" status code is returned in both cases. The user is informed of any problems using prominent human-readable error messages within the returned HTML page, but we would like these messages to be just as easy to find for machines.

We propose marking these error messages with the pmf-error semantic class name to make them trivial for software agents to find:

$<\mathrm{p}$ class="pmf-error" $>$ Incorrect username and/or password $</ \mathrm{p}>$

\section{Related work}

In parallel with our work on PMF, a proposal has been added to the HTML 5.1 working draft [6] which may achieve similar goals in future browsers. The HTML 5.1 draft specifies a new range of values for the "autocomplete" attribute for input elements. The autocomplete attribute disambiguates the semantic meaning of inputs in a similar way to our PMF semantic class names. It's not clear when this will be widely implemented but, in the meantime, our simple solution can be adopted immediately without compatibility concerns, since an input element could specify an autocomplete attribute value as well as a PMF semantic class name.

The innovative Stanford password policy of 2014 [11] is length-dependent: it imposes no restrictions on the characters of passwords above a certain length (20 characters), while prescribing stricter and stricter composition rules for progressively shorter passwords, and forcing a minimum length of 8 characters. It would already be PMF-compliant.

Bonneau and Preibusch's [10] comprehensive review of the authentication landscape on the web argues that some sites deploying passwords do so primarily for psychological rather than security reasons. For example, they speculate that password-protecting accounts serves as a justification for collecting marketing data and as a way to build trusted relationships with customers. Whatever the underlying reasons, it is apparent that the number of password-protected accounts an average user manages has increased markedly since the advent of the web. Florencio and Herley [12] report that the average user has 6.5 passwords, each of which is shared across 3.9 different sites. Furthermore, each user

$\overline{10}$ Note how our new requirement of adding the "if length $>t$ " statement to the policy may at some level represent a more significant change to the website but in practice involves much less work than our old requirement of accurately expressing the existing password composition policy in machine-readable form. 
has about 25 accounts that require passwords. Without the reported level of password reuse, managing 25 separate accounts with unique random passwords is barely imaginable for most users.

A password manager, either as a separate program such as PasswordSafe [13] or integrated with or in the browser, is now a well established solution for managing the increasing burden password-based authentication on the web. Given the increasing reliance on password managers ${ }^{11}$, a recent thread of research has investigated their security properties.

Gasti and Rasmussen [14] investigate the security properties of the password database formats used in range of popular password managers. They define two new games to analyse the security of password manager databases: indistinguishability of databases (IND-CDBA) game and chosen database (MAL-CDBA) game; the indistinguishability of databases game models the capabilities of a realistic passive adversary, and the chosen database game models the capabilities of an active adversary able to both read and write the password database file. Google Chrome stores plaintext username/passwords in the user's profile directory. As a result, an attacker can trivially win both the IND-CDBA and MALCDBA games with Chrome as the Challenger. Firefox also fails both games; however, Firefox optionally allows users to encrypt the passwords stored in the password managers database under a user-supplied master key. This option provides at least some security benefits over Google Chrome's password manager, even if the full benefits of indistinguishability under the IND-CDBA and MALCDBA games aren't afforded. Gasti and Rasmussen's analysis concludes that, among the systems they studied, only PasswordSafe v3 [13] is invulnerable to attackers under the IND-CDBA and MAL-CDBA security models.

Silver et al [15] identify a class of vulnerabilities exploitable when using several popular passwords managers. They consider a threat model where a user connecting to the Internet via an access point, such as a WiFi hotspot, which is controlled by the attacker. Under this model the attacker is able to inject, block and modify packets on the network. The attacker's goal is to extract passwords stored by the password manager without further action from the user. The attacks presented by Silver et al rely on exploiting the password manager auto-filling policies: for example, the password manager can be coerced into autofilling forms in invisible iframes embedded within the WiFi hot-spot's landing page. $^{12}$

Li et al [16] analysed the security of five popular integrated password managers (that is, password managers integrated with or in the web browser). Four key concerns with browser-based password managers were identified in this

$\overline{11}$ As an example, 1Password alone is estimated to have a install base of 2 to 3 million users.

12 Auto-filling of forms by the password manager improves usability and therefore, before mitigating this vulnerability by disabling the auto-filling, careful consideration is needed of the inherent trade off between security and usability. We shouldn't lose sight of the fact that normal users don't have threat models; therefore, simply asking them whether they want to enable or disable auto-filling is a bit of a cop out. 
study: bookmarklet vulnerabilities, web vulnerabilities, authorisation vulnerabilities and user interface vulnerabilities. Bookmarklet ${ }^{13}$ vulnerabilities, introduced by Adida et al [17], result from the bookmarklet's code running in a JavaScript environment potentially under the control of an attacker. Li et al show that such vulnerabilities are still widespread in popular password managers. The web vulnerabilities identified by $\mathrm{Li}$ et al consist of well know cross-site request forgery (CSRF) and cross-site scripting (XSS) attacks. The authorisation flaws identified by Li et al result from sloppy implementations. User interface vulnerabilities can be considered as phishing attacks against the password manager itself. In cases where the user is not authenticated to their password manager, a number of in-browser password managers automatically open the login form for the password manager in an iframe. Users have no means to differentiate between this behaviour and a phishing attack.

Password managers should be considered as tactical solutions, alleviating some of the gross security and usability failings of passwords. Pico [18] is a strategic solution seeking a more usable and secure replacement for passwords everywhere they are used (not just on the web). Recent work on Pico has attempted to provide a mechanism that can work alongside passwords [19]. The Pico bootstrapping technologies, whilst not being a password manager in the classic sense, are required to parse and automatically submit login forms on the user's behalf and would thus also benefit from our semantic annotations.

\section{Conclusions}

All password managers rely on fallible heuristics and complex code which is never fully accurate and requires constant updates. This entails wasteful replication of efforts by every password manager developer and reduces the value of password managers to users. We argue that all parties would benefit if websites offered a standard interface to password managers, enabling consistent and accurate agent-supported password creation, registration and login, without brittle programmatic guesswork.

Our PMF proposal, of augmenting a website's password pages with simple and unambiguous machine-readable semantics, makes the operation of password managers much simpler and more reliable. Users benefit from reduced cognitive load and reduced typing burden. Reliable generation of strong random passwords increases security for both users and websites. A well-defined interface eliminates guesswork and makes the password manager code leaner and much easier to maintain. We feel PMF is beneficial for all parties involved: users, website operators, password manager developers. We will be pleased to work with developers of websites, browsers and password managers, as well as with standards bodies, to promote its widespread adoption.

$\overline{13}$ A bookmarklet is a bookmark containing JavaScript that can be used to extend a web browser's capabilities. Bookmarklets have advantages over alternatives such as addons or extensions as they are cross browser and are managed by the user like bookmarks. 


\section{Acknowledgements}

We gratefully acknowledge the European Research Council for funding this research under grant 307224 (Pico).

\section{References}

1. Pinterest. https://pinterest. com Accessed: 2014-11-07.

2. OWASP: Cross-site request forgery (csrf) prevention cheat sheet. https:///www . owasp.org/index.php/Cross-Site_Request_Forgery_\%28CSRF\% 29_Prevention_Cheat_Sheet (August 2014) Accessed: 2014-11-06.

3. Beautement, A., Sasse, M.A., Wonham, M.: The compliance budget: Managing security behaviour in organisations. In: Proceedings of the 2008 Workshop on New Security Paradigms. NSPW '08, New York, NY, USA, ACM (2008) 47-58

4. Hickson, I., Berjon, R., Faulkner, S., Leithead, T., Doyle Navara, E., O'Connor, E., Pfeiffer, S.: HTML5. Recommendation, W3C (October 2014)

5. Stuven, Sybrel (W3C): Use class with semantics in mind. http://www.w3.org/ QA/Tips/goodclassnames Accessed: 2014-11-07.

6. Berjon, R., Faulkner, S., Leithead, T., Doyle Navara, E., O'Connor, E., Pfeiffer, S., Hickson, I.: HTML 5.1. Working draft, W3C (2014)

7. Hardt, D.: The OAuth 2.0 Authorization Framework. RFC 6749 (Proposed Standard) (October 2012)

8. Django documentation: Cross Site Request Forgery protection. https://docs . djangoproject.com/en/1.7/ref/contrib/csrf/ Accessed: 2014-11-07.

9. Bonneau, J., Xu, R.: Of contraseñas, sysmawt, and mìmǎ: Character encoding issues for web passwords. In: Web 2.0 Security \& Privacy. (May 2012)

10. Bonneau, J., Preibusch, S.: The password thicket: technical and market failures in human authentication on the web. In: WEIS 2010. (2010)

11. Stanford University. https://itservices.stanford.edu/service/accounts/ passwords/quickguide Accessed: 2014-11-07.

12. Florencio, D., Herley, C.: A large-scale study of web password habits. In: Proceedings of the 16th International Conference on World Wide Web. WWW '07, New York, NY, USA, ACM (2007) 657-666

13. Schneier, B.: Password safe. https://www.schneier.com/passsafe.html Accessed: 2014-11-06.

14. Gasti, P., Rasmussen, K.B.: On the security of password manager database formats. In: ESORICS. (2012) 770-787

15. Silver, D., Jana, S., Boneh, D., Chen, E., Jackson, C.: Password managers: Attacks and defenses. In: 23rd USENIX Security Symposium (USENIX Security 14), San Diego, CA, USENIX Association (August 2014) 449-464

16. Li, Z., He, W., Akhawe, D., Song, D.: The emperor's new password manager: Security analysis of web-based password managers. In: 23rd USENIX Security Symposium (USENIX Security 14), San Diego, CA, USENIX Association (August 2014) 465-479

17. Adida, B., Barth, A., Jackson, C.: Rootkits for javascript environments. In: Proceedings of the 3rd USENIX Conference on Offensive Technologies. WOOT'09, Berkeley, CA, USA, USENIX Association (2009) 4-4

18. Stajano, F.: Pico: no more passwords! In: Proceedings of the 19th international conference on Security Protocols. SP'11, Berlin, Heidelberg, Springer-Verlag (2011) $49-81$ 
19. Stajano, F., Jenkinson, G., Payne, J., Spencer, M., Stafford-Fraser, Q., Warrington, C.: Bootstrapping adoption of the pico password replacement system. In Christianson, B., Malcolm, J.A., Matyás, V., Svenda, P., Stajano, F., Anderson, J., eds.: Security Protocols XXII - 22nd International Workshop Cambridge, UK, March 19-21, 2014 Revised Selected Papers. Volume 8809 of Lecture Notes in Computer Science., Springer (2014) 172-186 